\title{
The Effects of Resistance Training with Palm Pollen on Scleraxis Protein and Gene Expression Levels in the Tendon Tissue of Male Adult Rats
}

\author{
Mohammad Mousae', Mohammad Ali Azarbayjani ${ }^{1 *}$, Maghsoud Peeri ${ }^{1}$, Seyed Ali Hosseini² \\ 1. Department of Sport Physiology, Central Tehran Branch, Islamic Azad University, Tehran, Iran \\ 2. Department of Sport Physiology, Marvdasht Branch, Islamic Azad University, Marvdasht, Iran
}

\section{Article Type: \\ Original Article \\ Article History:}

Received: 25 Feb 2019

Revised: 20 Jul 2019

Accepted: 29 Sep 2019

\section{*Correspondence:}

Mohammad Ali Azarbayjani, Department of Sport Physiology, Central Tehran Branch, Islamic Azad University, Tehran, Iran ali.azarbayjani@gmail.com

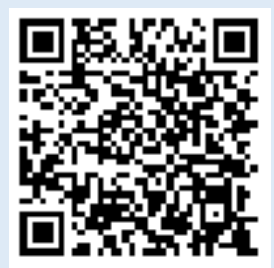

\begin{abstract}
Background and objectives: Controlling nutrition and exercise can be two important strategies in controlling tendon health. It has been reported that resistance training and palm pollen separately can improve Scleraxis (Scx) in tendon tissue; so present study aimed to investigate the interactive effects of resistance training with ethanolic extract of palm pollen on Scx protein and gene expression levels in the tendon tissue of male adult rats.

Methods: In this experimental study 30 male adult rats divided into 6 groups of 6 rats including: 1) sham, 2) training, 3) palm pollen, 4) testosterone, 5) training + palm pollen, and 6) training + testosterone. During 4 weeks, groups 2, 5, and 6 performed resistance trainings for five sessions per week; groups 3 and 5 received $100 \mathrm{mg} / \mathrm{kg}$ palm pollen for five days per week via gavage and groups 4 and 6 received $2 \mathrm{mg} / \mathrm{kg}$ testosterone propionate peritoneally. Scx protein and gene expression levels were measured in tendon tissue by Western blot and real-time PCR methods respectively. Shapiro- Wilk, one way ANOVA with Tukey's post- hoc tests were used to analyze the findings $(\mathrm{P} \leq 0.05)$.

Results: Training significantly increased Scx protein levels $(\mathrm{P}=0.005)$; palm pollen significantly increased Scx gene expression levels $(\mathrm{P}=0.001)$; training + palm pollen significantly increased Scx protein and gene expression levels $(\mathrm{P}=0.001)$ also training + palm pollen had more favorable effect on increase of Scx protein and gene expression levels compared to training and palm pollen alone $(\mathrm{P}=0.001)$.

Conclusion: It seems that resistance training simultaneously with palm pollen administration can have a more favorable effect than each one alone on improving Scx protein and gene expression levels in tendon tissue of male adult rats.
\end{abstract}

Keywords: Exercise; Phoeniceae; Tendons; Scx

Copyright $\subseteq$ 2018, Jorjani Biomedicine Journal has published this work as an open access article under the terms of the Creative Commons Attribution License (http://creativecommons.org/licenses/by-nc/4.0/) which permits noncommercial uses of the work while it is properly cited. 


\section{Introduction}

The knee is the most common site of osteoarthritis after the fingers and vertebrae, so that one-third of the world's population over the age of 65 is undergoing radiological changes in knee osteoarthritis. Studies have shown that the prevalence of knee osteoarthritis among people 65 years old and older is between 60 and 90 percent. This disease is one of the five most common causes of disability in non-hospitalized men and women (1). Proprioception sense in the joints leads to improved joint stability. Proprioception sense in the knee joint is caused by a combination of afferent messages from muscle receptors, tendons, joint capsules, ligaments, meniscus connections, and skin (2).

Due to their important role in the mechanical chain of the musculoskeletal system, tendons actually transmit muscle force to the bone levers. Since the tendon, as a regular dense connective tissue, has abundant collagen fibers along with fibroblast cells, on the other hand, due to the abundance of collagen fibers in the tendon, it is expected that by secreting and increasing the process of collagen production by fibroblasts, can accelerate the tendon healing. Tendons are known to be the densest connective tissue due to the superiority of connective fibers (collagen) over cellular and background material. They consist of packed parallel strands that place the background material in their very narrow space. Collagen filaments or primary tendon clusters are made of a large number of fibrils. The most abundant cell is the fibroblast or tendon cell, which is placed parallel to the longitudinal incisions between the collagen bands (2).
Tendons are damaged by various injuries and blows to the limbs. Factors that cause tendon damage include mechanical forces, minor injuries, and a series of blood supply and nutritional disorders. As tendon-feeding vessels are smaller than other connective tissues, the repair process in this tissue is slow. Due to the slow healing process of injuries in tendon, the selection of treatment technique should be based on a complete knowledge of the basic principles involved in the healing process of the tendon as well as the specific biomechanical conditions of this tissue (2). Scleraxis (Scx) is a DNA-binding transcription factor in the tendon and directly regulates the transcription of the type 1 collagen gene (which encodes many proteins in the tendon). So Scx is a key regulator of tenocyte differentiation, and its expression in tenocytes is highly due to TGF-beta (Tgfb) signaling. Decreased Tgfb and Scx indicate a disorder in tendon formation (3).

Studies have shown that two factors including exercise and proper nutrition are two strategies to prevent tendon tissue damage. In this regard, it has been reported that exercises can increase the stiffness and resistance of tendons (3). Also, sports activities can lead to the improvement of collagen levels (4-7). The use of medicinal plants to treat various diseases has been common in human societies since ancient times, and until about half a century ago, plants were one of the most important sources of medicine for the treatment of many diseases, and date is one of these plants. Date, scientifically known as phoenix dactylifera, is one of the most wellknown fruit trees for humans (8).

Palm pollen in date tree has been reported to increase plasma levels of testosterone and estradiol. Palm pollen also contains cholesterol, rutin and carotenoids, which 
causes the activity of gonadotropins in rats. Therefore, consumption of palm pollen can increase testosterone levels (9). Testosterone consumption has been shown to improve levels of tenocytes as well as types 1 and 2 collagen (10). Therefore, consumption of palm pollen seems to have similar effects to testosterone in strengthening and improving tendon tissue. Therefore, considering the supportive effects of exercises and palm pollen as well as lack of studies on the interactive effects of resistance training and palm pollen in tendon tissue, the present study aimed to investigate the effect of four weeks of resistance training and palm pollen on Scx protein and gene expression levels in the tendon tissue of male adult rats. Main hypothesis of present study is that the resistance training along with palm pollen has interactive effects on Scx protein and gene expression levels.

\section{Materials and Methods}

In this experimental study, 30 male adult rats were purchased and transferred to animal laboratory. The rats were kept under standard conditions (humidity of 45 to $55 \%$, dark-light cycle of 12-12 hours and temperature of $23 \pm$ $2 \circ \mathrm{C})$ for one week to adapt to the new environment with free access to food and water. In all periods of research all rats consumed standard food pellets including crude protein $23 \%$, crude fat $3.5 \%-4.5 \%$, crude fiber $4 \%-4.5 \%$, ash maximum $10 \%$, calcium $0.95 \%$ - $1 \%$, phosphorus $0.65 \%$ $0.75 \%$, salt $5 \%-5.5 \%$, humidity maximum $10 \%$, lysine $1.15 \%$, methionine $0.33 \%$, methionine + cysteine $0.63 \%$, threonine $0.72 \%$, and tryptophan $0.25 \%$. Then all rats divided into 6 groups of 6 rats including: 1) sham, 2) training, 3) palm pollen, 4) testosterone, 5) training + palm pollen, and 6) training + testosterone. During 4 weeks, groups 2, 5, and 6 performed resistance trainings for five sessions per week (11); groups 3 and 5 received $100 \mathrm{mg} / \mathrm{kg}$ palm pollen for five days per week via gavage (12) and groups 4 and 6 received $2 \mathrm{mg} / \mathrm{kg}$ testosterone propionate peritoneally (13). Forty- eight hours after the last training session as well as palm pollen and testosterone administration, the rats after 12 hours fasting were anesthetized with ketamine $(50 \mathrm{mg} / \mathrm{kg})$ (Sigma-Aldrich Company) and xylazine $\quad(10 \mathrm{mg} / \mathrm{kg}) \quad$ (Sigma-Aldrich Company), and after extracting tendon tissue and transferring it to laboratory; the Scx protein and gene expression levels were measured using Western blot and real-time PCR methods respectively. It should be noted that researchers received introduction letters from Marvdasht Branch of Islamic Azad University.

\section{Resistance training protocol}

To perform the resistance trainings, the rats were first introduced to climbing a ladder for one week. The weight of the rats was calculated, and the training program was adjusted based on the initial weight. In the first session, a weight equal to $50 \%$ of the weight of each rat was attached to the tail and the rats climbed the ladder. If the animal was able to climb, then 30 grams added to the weight and the animal climbed the ladder again. Also, in case of capability to lift 30 grams, extra weight was added to the tail. This was done as long as the animal was able to climb. The highest weight the animal could carry was the maximum strength value of the animal in the first session. In the next session, the rats performed 4 sets of climbing ladders, with the first set rising 50 percent with maximum strength, the second set 75 percent, the third set 90 percent, and the fourth set with 100 percent of maximum strength 
climbing the ladder. After the fourth set, in case of capability, 30 grams added to weights in each set and rat climbed the ladder. The program continued in one session until disability to climbing. At the beginning of each week, the rats performed trainings according to the maximum weight they had moved at the end of the previous week (11).

\section{Preparation of ethanolic extract of palm pollen}

Palm pollen powder was prepared exclusively from a palm tree from the palm groves of Shahdad city in Kerman province and was kept in the refrigerator all the time from the preparation of this powder to the extract. The extract was $90 \%$ ethanol and all stages of extraction were produced in the Medicinal Plants Research Institute of Jihad-eUniversity which is located in Kamalshahr in Alborz province. $250 \mathrm{~g}$ of palm pollen was poured into the percolator. Extraction was performed with $90 \%$ ethanol at $670 \mathrm{ml}$. This was repeated three times. The resulting extracts were concentrated in distillation and the solvent was completely removed. The yield of extract was $17.87 \%$. The extract was fed to rats 5 days per week at a dose of 100 $\mathrm{mg} / \mathrm{kg}(12)$.

\section{Testosterone administration}

Testosterone propionate, manufactured by Iran Hormone, was dissolved in dimethyl sulfoxide (DMSO) and peritoneally injected at a dose of $2 \mathrm{mg} / \mathrm{kg}$ as a positive control (13).

\section{Scx measurement by real-time PCR method}

For molecular analysis at the gene expression level, first, extraction of RNA from the tendon tissue was carried out according to the manufacturer's protocol; then drawing on the light absorbance property at wavelength of $260 \mathrm{~nm}$, the concentration and degree of purity of the RNA sample was quantitatively obtained using the following equation:

$\mathrm{C}(\mu \mathrm{g} / \mu \mathrm{l})=\mathrm{A} 260 \times \varepsilon \times \mathrm{d} / 1000$

After extracting RNA with high purity and high concentration from all of the samples, cDNA synthesis steps were taken according to the manufacturer's protocol, and then the synthesized cDNA was used for reverse transcription reaction. Initially, the designed primers for genes were examined, and then genes expressions were examined by quantitative q-RT PCR method. The sequence of forward- reverse primers of Scx gene is reported in Table 1.

Table 1. Sequence of Forward-Reverse Primers of Scx Gene in qRT-PCR Reaction

\begin{tabular}{|l|l|l|}
\hline Gene & Forward & Reverse \\
\hline Scx & 5' TCATCCCGACCGAGCCAGCA 3' $^{\prime}$ & 5' CCACAAGGTGCCAGGAATG 3' \\
\hline Gapdh & 5' CAT ACT CAG CAC CAG CAT CAC C 3' $^{\prime}$ & 5' CCGCAGGCTTCACCCACCAG 3' \\
\hline
\end{tabular}




\section{Scx measurement by Western blotting method}

Western blotting method was used to measure the Scx protein. The tissue was placed inside the Falcon test tube 15 and 200 grams of tissue was poured into it at a rate of $0.5 \mathrm{~g}$ of the single-phase sliding solution. To preserve tissue proteins, aprotinin was added to it and homogenized using a homogenizer for five minutes at a rate of $8,000 \mathrm{rpm}$. The resulting solution was centrifuged at $15 \mathrm{rpm}$ for 15 minutes. The upper solution was transferred into the microtubule by the sampler, and the remaining sediment was discarded. From the slipped tissue, the protein was then separated by SDS-PAGE using a $12 \%$ Tris-Glycine (Invitrogen) gel and the protein was tested using a Western blotting machine made by BioRad Company (made in UK). Western blotting using Scx antibody (Abcam; USA) (dilution 1: 1000) was performed, then reacted with the secondary HRP conjugated antibodies (Abcam; USA) (dilution 1: 2000). Finally, Blot was diagnosed using the ECL diagnostic system (Arlington, Amersham Life Science Inc., Arlington). Images obtained from the studied bands of each protein were analyzed using ImageJ software. To ensure equal amounts of protein at the time of measurement, the protein content was determined by lowry method before testing. GAPDH protein was used as an internal control in this study.

\section{Statistical analysis}

The Shapiro- Wilk test was used to investigate the normal distribution of the findings and one way ANOVA with Tukey's post- hoc tests were used in SPSS software to analyze the findings $(\mathrm{P} \leq 0.05)$.

\section{Results}

Scx gene expression and protein levels in six groups of study are reported in Figures 1 and 2 respectively. The results of one- way ANOVA test showed that there were significant differences in $\mathrm{Scx}$ protein $(\mathrm{P}=0.001)$ and gene expression $(\mathrm{P}=0.001)$ levels between six groups of study.

The results of Tukey's post- hoc test showed that Scx gene expression levels in palm pollen, testosterone, training + palm pollen and training + testosterone groups were significantly higher than sham group $(\mathrm{P}=0.001)$ also in training + palm pollen and training + testosterone groups were significantly higher than training, palm pollen and testosterone groups $(\mathrm{P}=0.001)$ (Figure 1). The results of Tukey's post- hoc test showed that $\mathrm{Scx}$ protein levels in training $(\mathrm{P}=0.005)$, testosterone $(\mathrm{P}=0.001)$, training + palm pollen $(\mathrm{P}=0.001)$ and training + testosterone $(\mathrm{P}=0.001)$ groups were significantly higher than sham group; in testosterone, training + palm pollen and training + testosterone groups were significantly higher than training and palm pollen groups $(\mathrm{P}=0.001)$; in training + palm pollen and training + testosterone groups were significantly higher than testosterone group $(\mathrm{P}=0.001)$ also in training + testosterone group were significantly higher than training + palm pollen group $(\mathrm{P}=0.002)$ (Figures 2 and 3). 


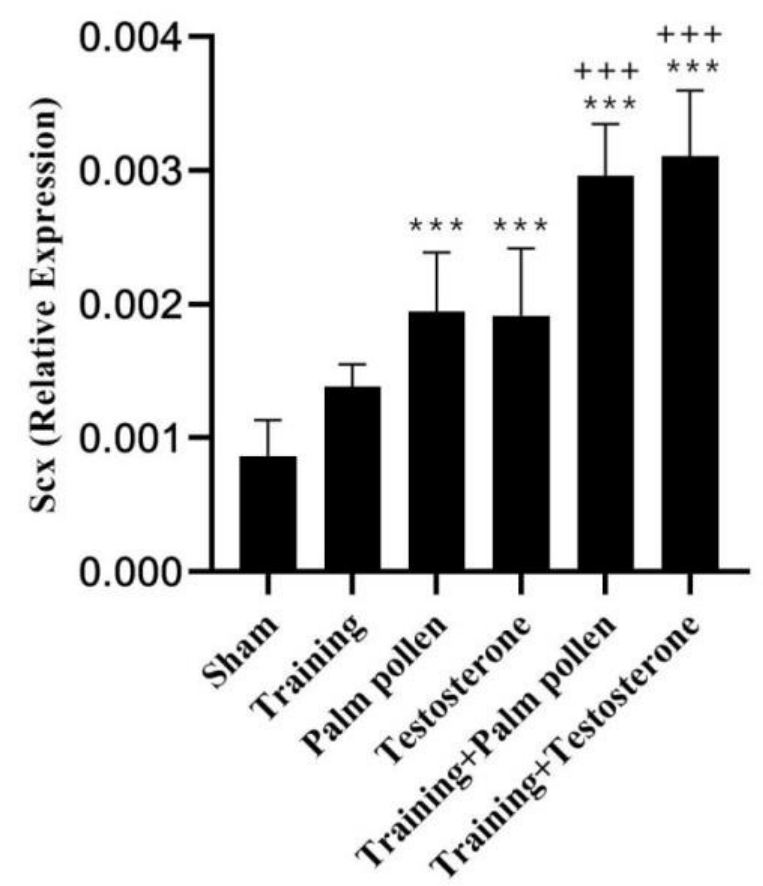

Figure 1. Scx gene expression levels in six groups of study

$* * * \mathrm{P}<0.001$ Significant increase compare to sham group; $+++\mathrm{P}<0.001$ Significant increase compare to training, palm pollen and testosterone groups

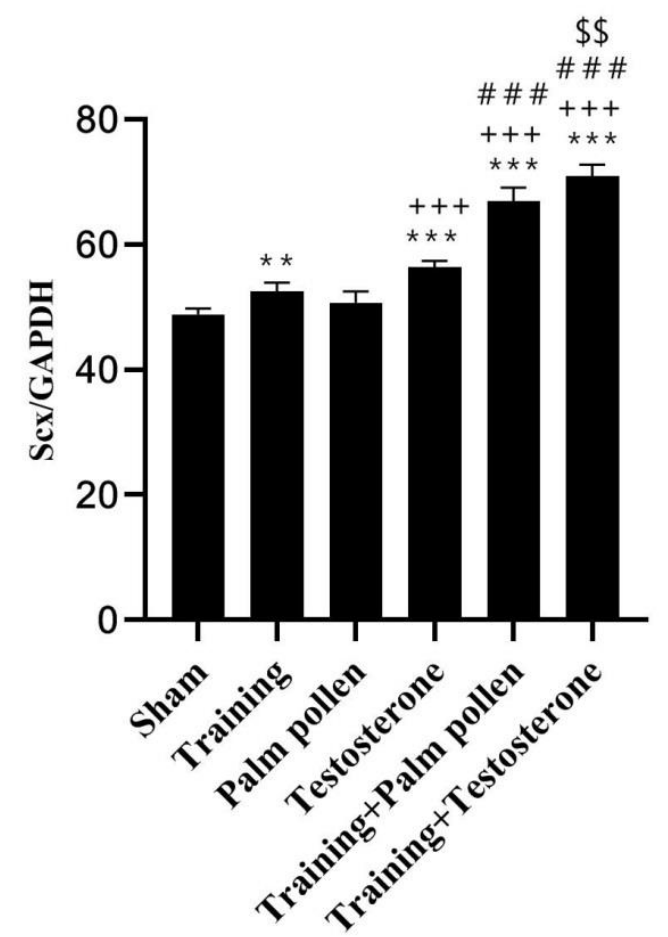

Figure 2. Scx protein levels in six groups of study

$* * * \mathrm{P}<0.001, * * \mathrm{P}<0.01$ Significant increase compare to sham group; $+++\mathrm{P}<0.001$ Significant increase compare to training and palm pollen groups; \# \# \# $<0.001$ Significant increase compare to testosterone group; $\$ \$ \mathrm{P}<0.01$ Significant increase compare to training + palm pollen group 

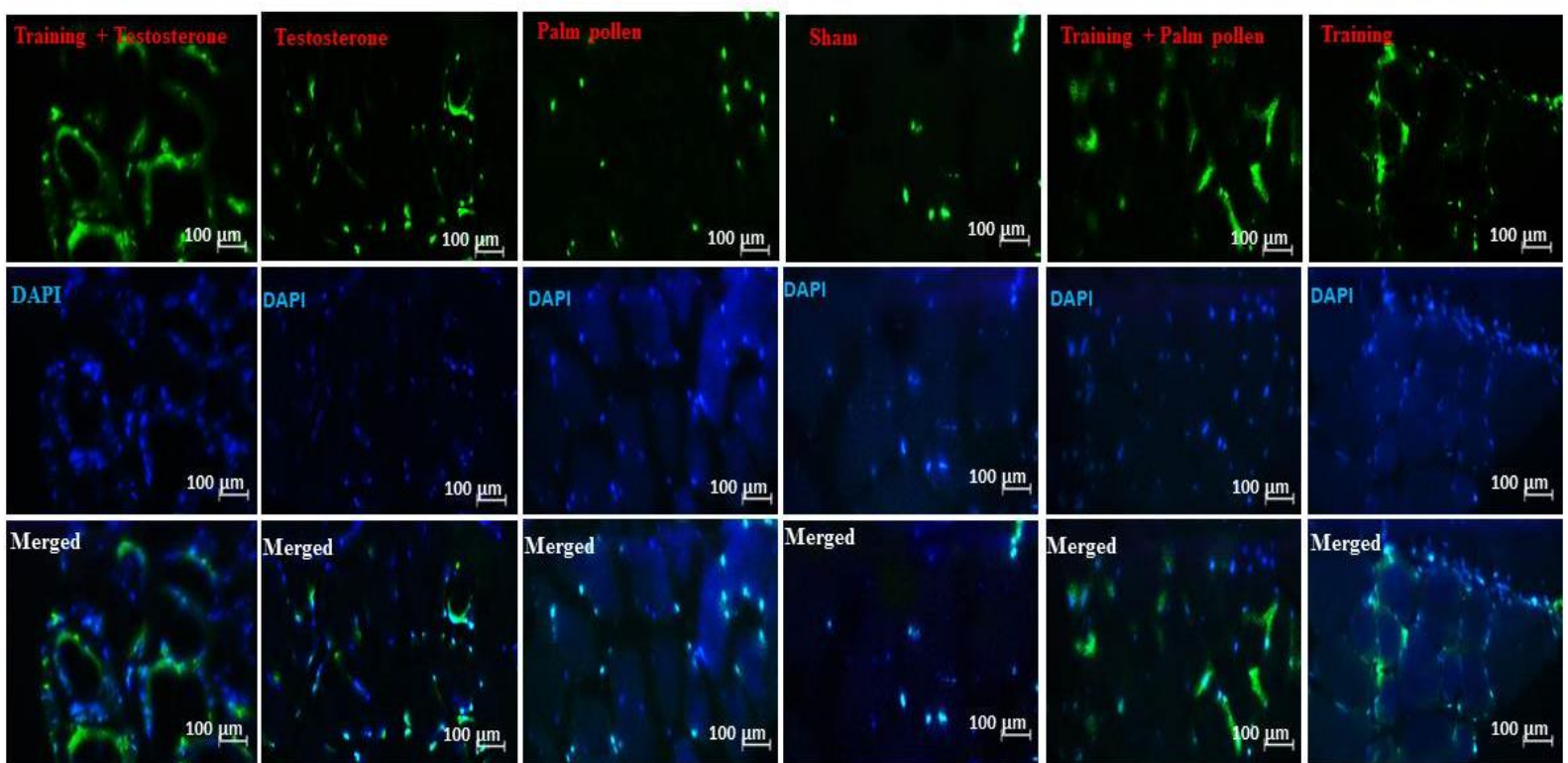

Figure 3. Immunohistochemical analysis of Scx marker in tendon tissues. The difference signs represent statistically significant differences between the mean values $(\mathrm{P}<0.001)$.

\section{Discussion}

The results of the present study showed that four weeks of resistance training significantly increased Scx protein levels in the tendon tissue of male adult rats; however, did not have a significant effect on Scx gene expression levels. Increases in several vasodilators (such as bradykinin and adenosine) have been reported in peritendinous tissue following exercise (14). Exercise has also been reported to increase blood circulation to the tendons three to seven times, and mechanical overload increases three to six times the oxygen uptake in the tendons (15). Therefore, it seems that exercises by increasing blood circulation and nutrient supply lead to improved tendon function and can increase the number of tendon-producing cells. Regarding to growth factors in animal studies, it has been shown that overload caused by exercises on the Achilles tendon leads to increased expression of IGF-1 and Tgfb, and these hormones are involved in the synthesis of exercise- induced collagen (14). Mechanical traction also increased mRNA levels of tenascin C, type 1 and 3 collagen and Scx in rats (4). Tendon repair occurs in three common stages. During the first stage, which is the inflammatory phase, monocytes and macrophages are the dominant cells and remove the damaged and dead tissue. Absorbent and vasoactive chemicals are released and stimulate the proliferation of fibroblasts and absorb inflammatory cells in the environment. In the second stage, which is the stage of cell proliferation, it begins 90 hours after the injury and lasts about a week. New vascular formation, proliferation of fibroblasts, collagen synthesis, and matrix formation occur at this stage. The third stage, in which regeneration takes place, is shared with the cell proliferation and growth stage and continues for a period of time, during which time collagen clusters are organized and regulated, and tissue and cell repair occurs (16).

The results of the present study showed that four weeks of palm pollen administration significantly increased Scx gene expression 
levels in the tendon tissue of male adult rats, so that palm pollen and testosterone (as positive control) had the same effects on increasing Scx gene expression levels. Both fresh and dried dates contain many nutrients, various sugars, non-fatty acids (saturated linoleic acid and oleic acid) and saturated fatty acids (acetic acid and palmitic acid), as well as about 15 minerals such as zinc, cadmium, calcium, potassium, vitamins A, C, $\mathrm{B} 1, \mathrm{~B} 2$ and niacin are other compounds in date (8). Research has shown that palm pollen contains estrogenic substances such as estrone and sterols as gonadal stimulants (9). In one study, it was shown that palm pollen consumption significantly increased estradiol, gonadotropin, and testosterone in male and female rats (17). The presence of compounds such as zinc and cadmium in palm pollen through increased biosynthesis of $17 \beta$ hydroxysteroid dehydrogenases increases testosterone production and steroid metabolism (8). Specialized testosterone receptors have been reported to be present in tendons. In vitro studies have shown that high concentrations of dihydrotestosterone increase the production of tenocytes in tendons; therefore, testosterone appears to lead to the differentiation of mesenchymal pluripotent cells in tendons (18). According to the above information, it appears that in present study palm pollen could increase the Scx gene expression levels in tendon tissue of male adult rats via increasing testosterone. Therefore, measuring serum testosterone levels in the present study could confirm the certainty of this theory.

Regarding to interactive effects, the results of the present study showed that resistance training simultaneously with palm pollen consumption significantly increased Scx protein and gene expression levels in the tendon tissue of male adult rats, so that resistance training simultaneously with palm pollen had more favorable effects compare to resistance training and palm pollen alone. These findings indicate the interactive and synergistic effects of resistance training and palm pollen consumption. As exercise strengthen the musculoskeletal-tendon receptors of the proprioception sense, including the muscular spindles and the Golgi- tendon apparatus as well as strengthen the orientation sense and sense of motion in joint also strengthen the primary cells of tendon via increasing blood circulation to the tendons; but palm pollen improves the function of the tendons by applying quasitestosterone effects, so it seems that exercise and palm pollen improve tendon function with separate mechanisms. Unable to measure serum testosterone levels and tissue levels of collagen with ELISA and western blotting methods were the research limitations of the present study, so in future studies, in addition to measuring serum testosterone levels and tissue levels of collagen, it is recommended to study the effect of resistance training with different intensities along with different doses of palm pollen on proliferation and differentiation of tenocyte cells.

\section{Conclusion}

It seems that resistance training simultaneously with palm pollen administration can have a more favorable effect than each one alone on improving Scx protein and gene expression levels in the tendon tissue of male adult rats. 


\section{References}

1. Akbarnajad N, Mohammadi Shahbolaghi F, Khankeh H R, Sokhangoiy Y, Biglaryan A, Sedghi N. Effect of Acupressure on knee pain and stiffness intensity in elderly patients with osteoarthritis: a randomized clinical trial. IJRN. 2016; 2 (2): 22-31.

2. Hosseini A, Ghorbanian M, Reza zadeh M, Azari A. Investigating the therapeutic role of low power laser beams on tendon rupture. Res Sport Sci. 2001; 1: 62-75.

3. Scott A, Danielson P, Abraham T, Fong G, Sampaio AV, Underhill TM. Mechanical force modulates scleraxis expression in bioartificial tendons. J Musculoskelet Neuronal Interact. 2011; 11 (2): 124-32.

4. Thorsen K, Kristoffersson A, Lorentzon R. The effects of brisk walking on markers of bone and calcium metabolism in postmenopausal women. Calcif Tissue Int 1996; 58: 221-5. [DOI:10.1007/BF02508639]

5. Langberg H, Skovgaard D, Asp S. Time pattern of exercise-induced changes in type I collagen turnover after prolonged endurance exercise in humans. Calcif Tissue Int 2000; 67: 41-4. [DOI:10.1007/s00223001094]

6. Tofas T, Jamurtas AZ, Fatouros I. Plyometric exercise increases serum indices of muscle damage and collagen breakdown. J Strength Cond Res 2008; 22: 490-6. [DOI:10.1519/JSC.0b013e31816605a0]

7. Brown S, Day S, Donnelly A. Indirect evidence of human skeletal muscle damage and collagen breakdown after eccentric muscle actions. J Sports Sci 1999; 17: 397-402. [DOI:10.1080/026404199365911]

8. Hosseini S E, Mehrabani D, Razavi F S, RafieiRad M. The effect of palm pollen aqueous extract on the sex ratio of offspring in mice strain BALB/c. Yafte J. 2013; 15 (2): 121-128.
9. Mahaldashtian M, Naghdi M, Ghorbanian M T, Koruji M, Makoolati Z, Naghizadeh M M, et al. The effect of aqueous extract of Phoenix Dactylifera Pollen on In vitro viability and proliferation rate of neonatal mouse spermatogonial stem cells. J Fasa Univ Med Sci. 2015; 4 (4): 402-408.

10. Abate M, Guelfi M, Pantalone A. Therapeutic use of hormones on tendinopathies: a narrative review. Muscles Ligaments Tendons J. 2016; 6 (4): 445-452. [DOI:10.11138/mltj/2016.6.4.445]

11. Hornberger TA Jr, Farrar RP. Physiological hypertrophy of the FHL muscle following 8 weeks of progressive resistance exercise in the rat. Can J Appl Physiol. 2004; 29 (1): 16-31. [DOI:10.1139/h04-002]

12. Mehraban F, Jafari M, Akbartabar Toori M, Sadeghi H, Joodi B, Mostafazade M et al. Effects of date palm pollen (Phoenix dactylifera L.) and Astragalus ovinus on sperm parameters and sex hormones in adult male rats. IJRM. 2014; 12 (10): 705-712.

13. Chodari L, Mohammadi M, Mohaddes G, Alipour MR, Ghorbanzade V, Dariushnejad H. Testosterone and voluntary exercise, alone or together increase cardiac activation of AKT and ERK1/2 in diabetic rats. Arq Bras Cardiol. 2016; 107 (6): 532-541. [DOI:10.5935/abc.20160174]

14. Langberg H, Bjørn C, Boushel R, Hellsten Y, Kjær M. Exercise induced increase in interstitial bradykinin and adenosine concentration of skeletal muscle and peritendinous tissue in humans. J Physiol. 2002; 542: 977-983. [DOI:10.1113/jphysiol.2002.018077]

15. Boushel R, Langberg H, Green S, Bülow J, Skovgaard D, Kjær M. Blood flow and oxygenation in peritendinous tissue and calf muscle during dynamic exercise in humans. $\mathbf{J}$ Physiol. 2000; 524: 305-313. [DOI:10.1111/j.1469-7793.2000.t01-2-00305.x]

16. Yahiae B. Histological study of the effect of ethanol on the injured tendon in rat. $\mathrm{J}$ Animal Biol. 2015; 7 (3): 73-80. 
17. El Mougy SA, Abed Aziz SA, Al-Shanawany M, Omar A. The gonadotropic activity of palmae in mature male rats. Alexandria J Pharm Sci. 1991; 5: 156-9.

18. Abate M, Guelfi M, Pantalone A. Therapeutic use of hormones on tendinopathies: a narrative

\section{How to cite:}

Mousae M, Azarbayjani M.A, Peeri M, Hosseini S.A. The Effects of Resistance Training with Palm Pollen on Scleraxis Protein and Gene Expression Levels in the Tendon Tissue of Male Adult Rats. Jorjani Biomedicine Journal. 2019; 7(4): 29-38. 